\title{
DATEV Design-DNA - Entwicklung einer produktübergreifenden Designsprache
}

\author{
Ulf Schubert \\ DATEV eG \\ Fürther Straße 111 \\ 90329 Nürnberg \\ ulf.schubert@datev.de
}

\section{Kristina Helmreich}

DATEV eG

Fürther Straße 111

90329 Nürnberg

kristina.helmreich@datev.de

\author{
Claude Toussaint \\ designaffairs \\ Balanstraße 73 I Haus 32 \\ 81541 München \\ claude.toussaint@designaffairs.com
}

\begin{abstract}
Die DATEV ist sich des Nutzens einer starken Marke bewusst. Sie entwickelt daher ihren Markenauftritt stringent und kontinuierlich weiter. Eine wesentliche Herausforderung dabei ist es, die Markenwerte an den zahlreichen Kontaktpunkten der Genossenschaft konsistent zu kommunizieren. Um dies zu gewährleisten, wurde in den letzten Jahren zusammen mit designaffairs eine Design-DNA konzipiert und eingeführt, welche die Klammer über alle Kontaktpunkte bilden soll. Wir beschreiben in diesem Beitrag, wie die Design-DNA konzipiert wurde, geben Einblicke in die Grundideen und erläutern die Herausforderungen bei der Einführung einer durchgängigen Designsprache in großen Unternehmen.
\end{abstract}

\section{Keywords}

Designstrategie, Designmanagement, Markenführung, Corporate Branding, Designsprache 


\section{Einleitung}

Die DATEV ist das Softwarehaus und der IT-Dienstleister für Steuerberater, Wirtschaftsprüfer und Rechtsanwälte sowie deren Mandanten. Als Genossenschaft besteht das wichtigste Ziel der DATEV in der wirtschaftlichen Förderung ihrer Mitglieder. DATEV unterstützt ihre Mitglieder bei deren beruflicher Tätigkeit durch Softwareprodukte und andere Dienstleistungen. (DATEV 2011) Das Streben nach höchster Qualität ist bei DATEV daher wichtiger als das Streben nach finanziellem Erfolg. Dies spiegelt sich auch im Markenauftritt der DATEV wider. Sie steht für Sicherheit (Datenschutz, Datensicherheit, Zukunftssicherheit), Erfolg (nachhaltige Förderung der Mitglieder, Leistung zum fairen Preis, bedarfsgerechte Software), Nähe (sowohl geografisch als auch im partnerschaftlichen Umgang) und Aktualität (aktuelle Software und Wegbereiter) (DATEV 2015).

\section{Ausgangssituation}

Ihren hohen Anspruch bzw. ihr Versprechen kommuniziert DATEV über einen stringent geführten Markenauftritt. Die DATEV zeichnet sich seit Jahren durch eine sehr fortschrittliche Markenführung aus. Spürbar und erlebbar wird dies an den zahlreichen Kommunikations-Kontaktpunkten von digitalen Medien über Druckerzeugnisse bis zu Veranstaltungen. In den letzten Jahren wurde die Markenführung immer weiter auch in den Bereich der Produktgestaltung ausgedehnt. Hier besteht die Herausforderung darin, die Markenwerte bzw. das Markenversprechen auch über die mehr als 200 Produkte der DATEV, die seit der Gründung der DATEV im Jahr 1966 entstanden sind und ständig weiterentwickelt wurden, zu kommunizieren.

Zum einen kommunizieren DATEV-Produkte die Markenwerte natürlich über ihre fachlichen und technischen aber auch über ihre gestalterischen Qualitäten und Eigenschaften. Der rasante technologische Fortschritt stellt die DATEV dabei vor große Herausforderungen. Wie kann es bei der Vielzahl der technischen Plattformen, Geräte und Ökosysteme in der digitalen Welt gelingen eine Designsprache $\mathrm{zu}$ entwickeln, die produktübergreifend eingesetzt werden kann und welche die Markenwerte an allen Punkten, an denen ein Anwender mit einem Produkt oder Anwendung in Kontakt kommt, konsistent kommuniziert? Wie kann es gelingen, mit einer Designsprache zwar eine konsistente Gestaltung über die mehr als 200 Produkte der DATEV zu erreichen, aber noch genügend Freiraum für die fachlichen, technischen bzw. zielgruppenspezifischen Anforderungen zu lassen? Wie kann es bei einem über 1.800 Mitarbeiter starken Entwicklungsteam gelingen, diese Designsprache zu etablieren?

\section{Ganzheitlicher Blick auf UX Design}

Bei der Betrachtung der Herausforderungen wurde schnell klar, dass es nicht genügt, einfach ein paar gestalterische Vorgaben $\mathrm{zu}$ machen und diese in einem Styleguide $\mathrm{zu}$ dokumentieren. Um diesen Herausforderungen begegnen zu können, war eine ganzheitliche 
und strategische Betrachtungsweise notwendig. Daher stand vor der Entwicklung der DATEV Design-DNA die Entwicklung einer Designstrategie. Mit dem Begriff „DATEV Design-DNA“ bezeichnen wir den Kern unserer Designsprache. Die DATEV Design-DNA umfasst alle wesentlichen Merkmale, die zur Kommunikation der Markenbotschaft und zur Gestaltung einer positiven User Experience eingesetzt werden.

Grundgedanke einer Designstrategie ist, dass die Produktgestaltung einen wesentlichen Beitrag zur Erreichung der Geschäftsziele eines Unternehmens liefern muss. Es ist eine Abkehr von dem in der Softwareentwicklung historisch weitverbreiteten Verständnis, dass die Produktgestaltung dazu dient, die Produkte durch einen modernen „Anstrich“ dem Kunden „schmackhafter“ zu machen. Eine Designstrategie beschreibt viel mehr, mit welchen gestalterischen Mitteln und Methoden die Erreichung bestimmter Unternehmensziele am besten unterstützt werden kann. Gestalterische Mittel können z.B. eine Designsprache oder Designprinzipien sein. Gestalterische Methoden sind beispielsweise Methoden der anwenderorientierten Gestaltung (Human Centered Design). Eine Designstrategie basiert immer auf den Geschäfts- und Produktstrategien eines Unternehmens und steckt den gestalterischen Rahmen für die mittel- bis langfristige Weiterentwicklung der Produkte ab. Da sich eine Designstrategie immer auch an den Bedürfnissen des Marktes sowie der Kunden orientieren muss und sich im Wesentlichen damit beschäftigt, wie die Lösungen für die Kunden- und Marktanforderungen aussehen sollen, bildet sie eine gute Grundlage für den Innovationsprozess.

Ein weiteres Ziel einer Designstrategie ist es, einen positiven Beitrag zur Erzeugung einer klaren und vorteilhaften Corporate Identity zu leisten. Dazu muss sie in Einklang mit dem Kommunikationsdesign, dem Environment-Design und der Unternehmenskultur gebracht werden. (Sommerlatte, 2009)

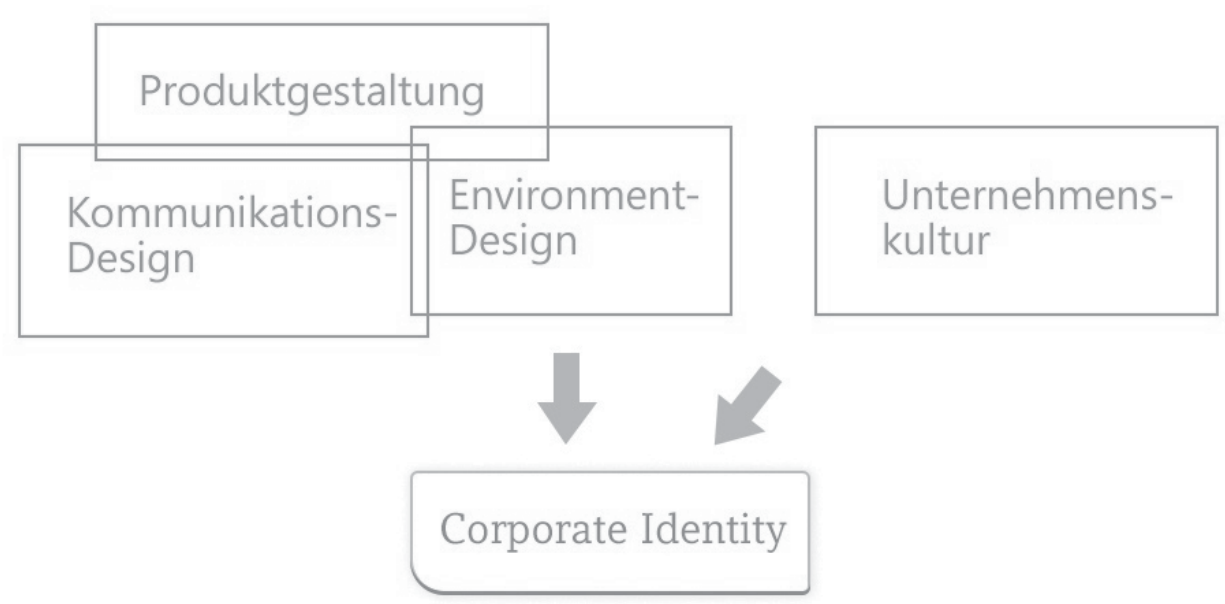

Abbildung 1: Zusammenspiel der Designdisziplinen nach Sommerlatte 
Mit Kommunikationsdesign ist die Gestaltung aller Kommunikationsinhalte eines Unternehmens gemeint. Dazu gehören neben der Marke auch die Kommunikationsmittel im Bereich des Marketings. Unter Environment-Design wird die Gestaltung der physischen Umgebung eines Unternehmens verstanden. Dazu gehört beispielsweise die Gestaltung der Arbeitsumgebung und der Gebäude eines Unternehmens. Nur ein Unternehmen, welches in allen vier Bereichen - Produktgestaltung, Kommunikationsdesign, Environment-Design und Unternehmenskultur - die gleichen Inhalte und Aussagen kommuniziert, kann die Vorteile einer ganzheitlichen Corporate Identity nutzen, wie z.B. Kundenbindung und Absatzförderung.

Im Vorfeld der Entwicklung der Designsprache wurde daher eine umfangreiche Analyse der Produktgestaltung, der Designorganisation und der gestaltungsrelevanten Trends aus Gesellschaft und Technik vorgenommen. Aus dieser Analyse wurden Handlungsfelder und Rahmenbedingungen für die DATEV Designstrategie abgeleitet. Es wurde bereits zu diesem frühen Zeitpunkt definiert, welche Themen bearbeitet, welche Voraussetzungen geschaffen und welche Veränderungen in der Organisation herbeigeführt werden müssen, um eine Designsprache erfolgreich einführen zu können.

\subsection{Entwickler, 200 Produkte, 1 Designsprache}

\section{Definition der grundlegenden Designrichtung}

Der erste Schritt bei der Entwicklung der DATEV Design-DNA war die Identifikation der Markenwerte, die am Kontaktpunkt „Produkt“ kommuniziert werden sollen. Kernfragen der durch designaffairs durchgeführten Markenanalyse waren daher: Was sind die primären Markenwerte? Wie können diese Werte visuell auf die Designsprache übertragen werden?

designaffairs analysierte mithilfe des neurosemantisches Analyse-Tools SimuPro® die DATEV Markenwerte. SimuPro ${ }^{\circledR}$ macht sichtbar, ob die gestalterischen Grundkonzepte zur Zielgruppe, Marke oder den Unternehmenswerten passen. Dazu werden die Markenwerte über semantische Differenziale auf allgemeingültige psychologische Werte übertragen. Das Ergebnis wird grafisch in dem SimuProß-Profil dargestellt. 


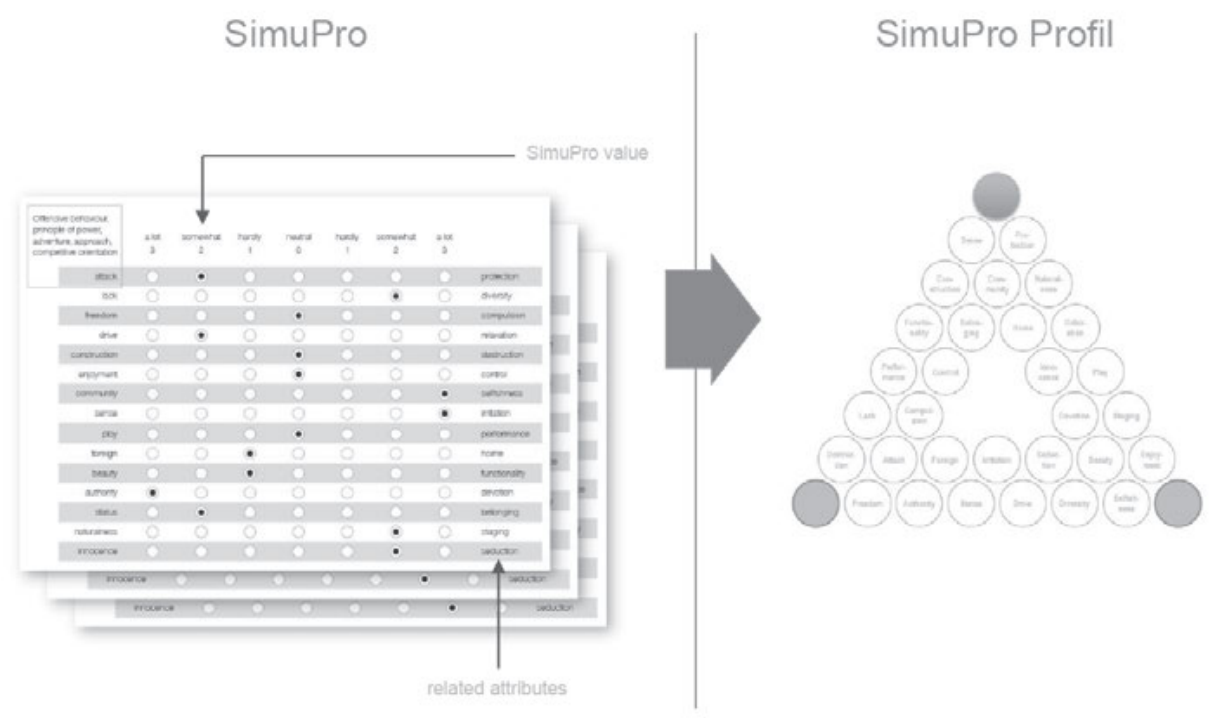

Abbildung 2: Neurosemantisches Tool „SimuPro®“zur Markenanalyse

designaffairs beobachtet seit vielen Jahren Designstile, beschreibt diese und bewertet diese mit Hilfe des SimuPro®. Im Laufe der letzten 10 Jahre wurde mit über 3.000 Probanden die Wirkung der relevantesten Designstile gemessen und eine große Datenbasis für Vergleiche geschaffen. Hierdurch war es möglich, auf Basis der identifizierten Markenwerte zuverlässig zu ermitteln, welcher grundlegende Designstil am besten für die Kommunikation der Markenwerte der DATEV geeignet ist. Der identifizierte Designstil beschreibt grob, über welche grundlegenden gestalterischen Merkmale die Produktgestaltung definiert werden soll.

\section{Kulturelle Übersetzung}

Ein abstrakter Designstil ist jedoch nicht genug, um als Leitlinie für die Gestaltung von zahlreichen Produkten durch eine große Entwicklungsorganisation zu dienen. Er ist zu generisch, reflektiert die Marke zu wenig und lässt zu viel Raum für Interpretation. Außerdem ist es sehr unwahrscheinlich, dass ein generischer Designstil - auch wenn er mit einem zuverlässigen Verfahren ermittelt wurde - von einer großen Entwicklungsorganisation als neue Designsprache einfach so akzeptiert und umgesetzt wird.

Grundidee des weiteren Vorgehens war daher, dass die neue Designsprache auf einer gemeinsam getragenen UX-Vision basieren sollte. Die UX-Vision sollte zur Definition der Ausrichtung und des Entscheidungsspielraums für die Produktgestaltung dienen. Sie soll dabei aber nicht nur regulierend, sondern auch inspirierend sein und als Messlatte für Designentscheidungen dienen. (Schubert, 2015) Damit eine UX-Vision ihre Wirkung entfalten kann, muss sie so entstehen, dass sie von Anfang an von allen wichtigen Beteiligten getragen und von diesen im Unternehmen vertreten wird. Eine UX-Vision funktioniert dann gut, wenn sie von vielen Personen im Unternehmen verinnerlicht wird. Dazu muss sie nicht 
nur sehr einfach sein, sondern auch von vielen mit Überzeugung wiederholt kommuniziert werden. Sie muss entsprechend fokussiert und einprägsam sein.

Daher wurde der Designstil mit Vertretern des mittleren Managements im Rahmen eines UX-Vision Workshops in UX-Prinzipien übersetzt. Es wurden bewusst Vertreter des mittleren Managements gewählt, da diese sowohl nah genug an der konkreten Umsetzung der Produkte beteiligt sind als auch über die notwendigen Entscheidungsfreiräume verfügen. Im UX-Vision Workshop wurden über die Bewertung und Gruppierung von Fotos erst Adjektive und dann Slogans abgeleitet. Die Fotos wurden dafür so ausgewählt, dass sie Gegenstände aus unterschiedlichen Bereichen zeigten, die dem identifizierten Designstil entsprachen. Die Slogans wurden danach durch die Erstellung von fiktiven Produktverpackungen priorisiert. Die Slogans, die am höchsten priorisiert wurden, wurden im Anschluss in UX Prinzipien überführt. Weitere Details zum Workshop sind im UX-Blog beschrieben. (Schubert, 2015)

\section{Gestalterische Übersetzung}

Im nächsten Schritt übersetzte designaffairs die UX-Prinzipien auf Basis von realistischen fachlichen sowie technischen Szenarien in Gestaltungsentwürfe. Auf diese Weise konnte ermittelt werden, wie sich die UX-Prinzipien mit visuellen und interaktiven Gestaltungsmitteln wirksam übersetzen lassen. In einem iterativen Verfahren wurden durch ein gemeinsames Designteam von designaffairs und DATEV die Entwürfe so lange überarbeitet, bis sie in Form eines Design-Leuchtturms für die Ableitung der konkreten Gestaltungsmerkmale sowie für die interne Kommunikation verwendet werden konnten.
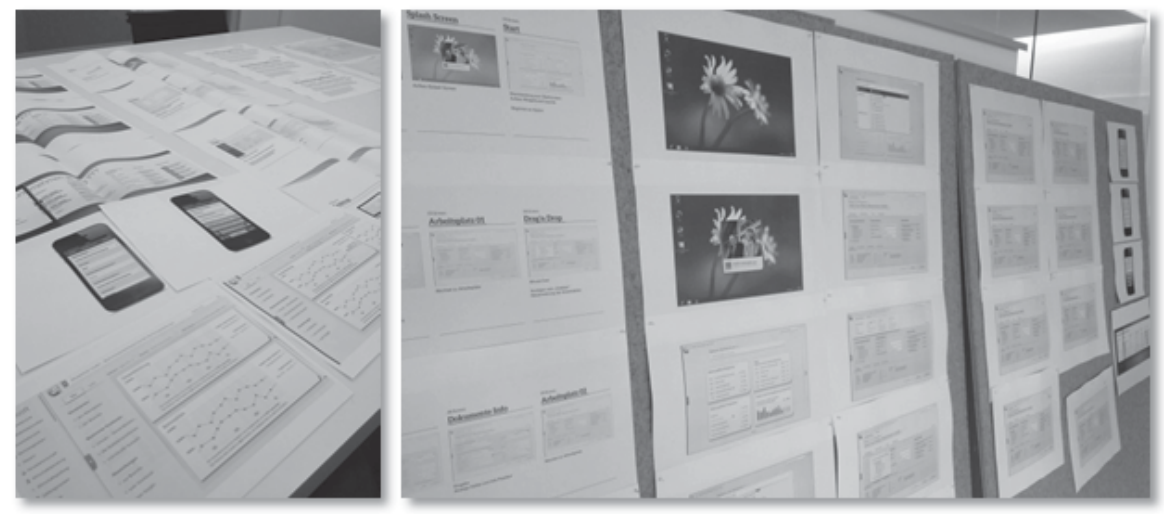

Abbildung 3: Konzeptionsphase der DATEV Design-DNA

Unter einem Design-Leuchtturm verstehen wir einen gestalterischen Prototypen, der visualisiert, wie sich ein Produkt mit einer bestimmten Designsprache anfühlt. Wie eine Art Concept Car aus der Automobilindustrie dient er als Basis für Richtungsentscheidungen bzgl. der Produktgestaltung sowie zur internen und externen Kommunikation. 


\section{Ableitung der Gestaltungsmerkmale}

Während des iterativen Erstellungsprozesses des Leuchtturms wurden Gestaltungsmerkmale als tragende Schlüsselelemente herausgearbeitet. Sie haben die Aufgabe, einen starken Eigencharakter und damit die Klammer zu bilden, die die Wiedererkennung über das Produktportfolio hinweg garantiert. Die Gestaltungsmerkmale wurden am Ende der Erstellung des Design-Leuchtturms dokumentiert. Für die DATEV Design-DNA wurden folgende Gestaltungsmerkmale definiert:

- Typographie: Eine typographische Kombination einer Serifen-Schrift und einer serifenlosen Grotesk-Schrift. Damit dieses Gestaltungsmerkmal seine Wirkung auch entfalten kann, wenn die definierten Schriften nicht verfügbar sind, wurden die Schriften so gewählt, dass für beide Schriften ähnliche Schriften auf allen technischen Plattformen vorhanden sind.

- Farbe: Ein markanter Farbdreiklang aus den Corporate Colors der Marke DATEV

- Icons: Ein Iconsystem, welches farblich und semantisch die Markenwerte transportiert. (Kirstein et.al., 2012)

- Form: Eine einfache und einprägsame Form.

- Animation: Eine Definition, wie Animationen umgesetzt werden sollen.

Im weiteren Verlauf der Etablierung der Designsprache wurden diese Gestaltungsmerkmale weiter verfeinert. Beispielsweise wurde das initial definierte Farbsystem durch die Umsetzung in diversen Entwicklungsprojekten erweitert und im Detail definiert.

Wie eingangs beschrieben, müssen die visuellen und interaktiven Gestaltungsmerkmale der DATEV Design-DNA auf der Vielzahl von technischen Plattformen und technischen Ökosystemen funktionieren. Um dies zu erreichen, wurden die Gestaltungsmerkmale nach der Prämisse konzipiert: die Gestaltungsmerkmale werden nur dann eingesetzt, wenn dies mit einem wirtschaftlich vertretbaren technischen Aufwand möglich ist und sie nicht im Widerspruch zu den Grundprinzipien der eingesetzten User Interface-Technologie stehen. Sie wurden daher so konzipiert, dass sie auch ihre Wirkung entfalten, wenn ein Gestaltungsmerkmal in einem Produkt nicht umgesetzt werden kann.

Eine weitere eingangs beschriebene Herausforderung war das Spannungsfeld zwischen einer einheitlichen Umsetzung der Designsprache in allen Produkten und dem Freiraum für fachliche, technische oder zielgruppenspezifische Anforderungen. Dieser Herausforderung begegnet die DATEV Design-DNA mit einer einfachen Antwort: der Reduktion auf die wesentlichen Gestaltungsmerkmale. Die DATEV Design-DNA umfasst nur die Gestaltungsmerkmale, die notwendig sind, um die Markenwerte zu kommunizieren. Sie ist bewusst einfach und reduziert, damit sie sich leicht einprägen und in der Organisation kommunizieren lässt. Sie bietet viel Freiraum für Kreativität und Innovation. Damit dieser Freiraum nachhaltig und im Sinne der Marke DATEV genutzt werden kann, wurde begleitend zur Einführung der DATEV Design-DNA eine Community of Practice (Wikipedia, 2015) für UX-Design etabliert. Die UX-Designer der Community arbeiten entweder im zentralen UX-Design-Team oder in einer produktentwickelnden Fachabteilung 
der DATEV. Auf diese Weise kann die Umsetzung der Designsprache in den Entwicklungsprojekten gesteuert werden. Es fließen aber auch Impulse aus den Entwicklungsprojekten für die Weiterentwicklung der Designsprache zurück.

\section{DATEV Design-DNA}

Zusammenfassend kann man sagen, dass die DATEV Design-DNA aus UX-Prinzipien und Gestaltungsmerkmalen besteht, welche die Grundlage für die zielgerichtete Kommunikation der Markenbotschaften über die Produktgestaltung bilden. Diese tragen dazu bei, dem Markenversprechen der DATEV gestalterisch in den Produkten gerecht zu werden.

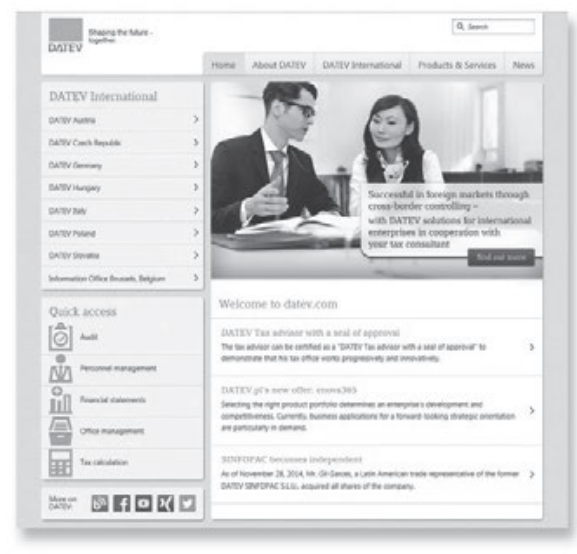

Abbildung 4: Umsetzung der DATEV Design-DNA in Beispielen

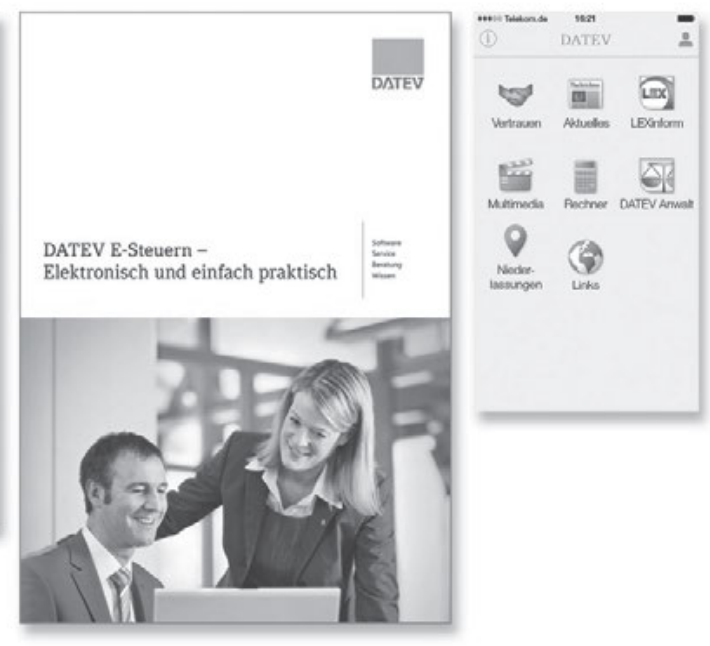

Durch die Herleitung über die Marke stellt die DATEV Design-DNA keinen isolierten Gestaltungsstil für Produkte dar. Sie bildet vielmehr eine Klammer über die verschiedenen Kontaktpunkte der Marke - vom Kommunikationsdesign über das Environment-Design bis hin zum Produktdesign.

\section{Literatur}

DATEV (2011). Satzung der Genossenschaft. Nürnberg: DATEV

DATEV (2015). Zwölf Gründe, sich für DATEV zu entscheiden. http://www.datev.de/portal/ShowPage.do?pid=dpi\&nid=120825

Kirstein, E., Schoenherr, N., Schubert, U. (2012). Icon Design im großen Stil - Erfahrungen zu Gestaltung und Einsatz von umfangreichen Icon Bibliotheken. In Brau, H. / et. al. (Hrsg.): „Usability Professionals 2012“, Berichtband der Usability Professionals / Mensch und Computer 2012, Konstanz, S. 60 
Schubert, U. (2015). Workshop-Format für die Entwicklung und Einführung einer UX-Vision. http://www.user-experience-blog.de/2015/02/ux-vision-gemeinsam-entwickeln/

Sommerlatte, T. (Hrsg.) (2009). Praxis des Designmanagements. Düsseldorf: Symposion Publishing

Wikipedia (2015). Community of Practice. http://de.wikipedia.org/wiki/Community_of_Practice 